\title{
st \\ Introdução a três textos de Einstein sobre a geometria, a teoria física e a experiência
}

\author{
Michel PATY
}

Os três artigos de Einstein publicados neste número são de natureza epistemológica e filosófica e estão diretamente vinculados a sua própria experiência na produção de conhecimentos da física teórica: eles constituem de certo modo uma espécie de momento reflexivo dessa experiência.

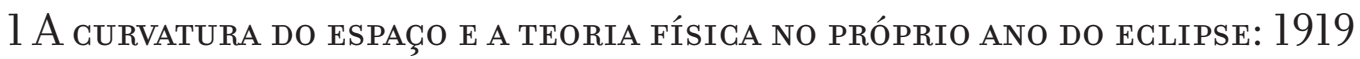

O primeiro texto, intitulado Indução e dedução na física (Einstein, 1919b), é um dos primeiros de natureza reflexiva e foi escrito em dezembro de 1919, cinco anos após o Discurso de recepção à Academia de Ciências da Prússia de 1914 que tratava dos princípios da física teórica (Einstein, 1914). Nele, Einstein apresenta brevemente, de modo "militante", como ele próprio enfatiza, suas concepções anti-indutivistas sobre a elaboração das teorias físicas. A teoria da relatividade geral, elaborada no mesmo decênio e publicada em 1915-1916, acabava de ser levada ao conhecimento de um público bastante amplo, em razão do anúncio recentíssimo dos resultados da observação astronômica da curvatura dos raios luminosos nas proximidades do Sol; resultados que a confirmavam de maneira espetacular (cf. Dyson, Eddington \& Davidson, 1920). Ainda que Einstein não lhe faça referência nesse artigo, a teoria, que começa então a torná-lo mundialmente célebre, e a experiência intelectual vivida da dificuldade de sua elaboração estão presentes em filigrana em suas primeiras considerações sobre o raciocínio hipotético-dedutivo e sobre o uso da intuição intelectual (com aquilo que ele chamará a "livre criação") na formação de conceitos e de teorias, idéias que constituirão o eixo central de sua epistemologia e que ele não deixará em seguida de aperfeiçoar. Encontram-se também indicações sobre o aspecto sistêmico das teorias, sobre o caráter provisório e perfectível de todo conhecimento e sobre a refutabilidade das teorias, formulada antes de Popper. 
Incidentalmente, pode-se perguntar por que esse artigo, publicado no grande jornal de Berlim, o Berliner Tageblatt, em 21 de dezembro de 1919, não foi retomado depois em obras de Einstein tais como Como vejo o mundo e Idéias e opiniões, que são coletâneas de seus artigos e conferências endereçadas ao grande público. Foi escrito um mês depois da publicação, no Times de Londres, do artigo Minha teoria, no qual Einstein fazia uma apresentação da relatividade geral (esse artigo foi republicado nos dois livros citados, sob o título $O$ que é a teoria geral da relatividade?). Ainda assim, o artigo Indução e dedução não foi totalmente ignorado. Ele é mencionado nas bibliografias mais completas dos escritos de Einstein (Shields, 1949; Paty, 1993, especialmente a Bibliografia), e uma tradução ao francês foi publicada no volume 5 das Oeuvres choisies, enquanto se espera a publicação iminente nos Collected papers.

Os dois escritos do final do ano de 1919 estão relacionados: um, Minha teoria, descreve as idéias-mestres da teoria de Einstein sublinhando sua natureza de teoria física (Einstein propõe nele sua distinção entre "teoria segundo princípios" e "teoria construtiva"), o outro, Indução e dedução, apresenta uma reflexão sobre a maneira pela qual o pensamento científico luta com o mundo para produzir dele uma representação, uma teoria, feita de princípios e de conceitos construídos, escolhidos pelo pensamento e provisórios, e sobre a maneira pela qual o pensamento se confronta com os dados da experiência. Esses dois artigos, que apareceram na grande imprensa, estão dentre os primeiros escritos de Einstein sobre questões dessa natureza e contêm a indicação de muitos dos temas dominantes de seu pensamento acerca da relação do mundo com o conhecimento que temos dele.

Pode-se fazer outra aproximação entre os dois artigos de jornal aparecidos ao final do ano da observação do eclipse solar: os dois terminam com uma alusão à situação política internacional da época. Em Minha teoria, Einstein indica que a elaboração (por ele mesmo) da teoria da relatividade geral e a realização (pelas expedições dirigidas por Eddington) de observações para testá-la foi a obra comum de cientistas pertencentes a nações que estavam então em guerra (respectivamente a Alemanha e a Inglaterra); ele sublinhava que essa cooperação de fato tinha podido transcender os conflitos e as paixões nacionais e exprimia assim seu vivo reconhecimento aos colegas ingleses. Evocava também, em uma observação acrescentada ao final do artigo, em tom irônico mas no fundo amargo, a "relatividade" dos juízos humanos em função da época e dos prejuízos que ela nutre. ${ }^{1}$

1 Einstein escrevia:

Observação. As observações de vosso jornal concernente a minha pessoa e as circunstâncias de minha vida são testemunho em parte da alegre imaginação do autor do artigo. Eis aí uma espécie de aplicação do princípio de relatividade para divertir o leitor: que eu seja hoje em dia chamado na Alemanha de “cientista alemão" e na Inglaterra de 
Introdução A três textos de Einstein...

Em Indução e dedução na física, Einstein se justifica por propor uma reflexão sobre as raízes do conhecimento fundamental, tão distante das preocupações quotidianas dos indivíduos e dos povos, em um jornal cujo conteúdo está essencialmente voltado para as preocupações sociais e políticas.

Nestes tempos instáveis, apresento ao leitor esta pequena reflexão, objetiva mas apaixonada, porque é minha opinião que, hoje em dia, é através da devoção silenciosa aos fins eternos comuns a todas as pessoas instruídas que posso ajudar na recuperação política, de uma maneira mais eficiente do que através de considerações e manifestações políticas.

Com efeito, nesses anos da República de Weimar que esperava construir uma democracia sobre as ruínas do Reich alemão destruído ao final da Primeira Guerra Mundial, Einstein se fez o embaixador da reconciliação entre os povos em nome da cultura e da ciência. (Foi nesse papel que ele participou da Comissão cultural da Sociedade das Nações e que o vemos viajar de trem de Berlim até Genebra em junho de 1925, tal como Norbert Wiener o descreve em uma carta a sua irmã, a qual também publicamos aqui.) $)^{2}$

Apesar do primeiro texto de Einstein que publicamos não abordar explicitamente a teoria geral da relatividade nem a questão da geometria, que é o objeto dos outros dois textos publicados a seguir, ele não lhes é estranho, já que sua teoria, e com ela a questão da geometria, são subjacentes a sua tomada de posição contra a indução e o empirismo e a favor da criação racional pelo pensamento. Como veremos, ele até mesmo esclarece antecipadamente suas conclusões.

\section{Dois teXtos ePISTEMológigos SObRe A GEOMETRIA: 1921 e 1926}

Os outros dois textos tratam da questão da natureza da geometria e de sua relação com a física e a experiência. Geometria e experiência é um texto hoje em dia clássico, freqüentemente citado, de certo modo histórico, pois é um elemento importante do debate sobre a questão da natureza da geometria e de sua relação com o mundo físico, a qual ocupou bastante os espíritos ao longo de todo o século xx. Após a descoberta das geometrias não-euclidianas no século XIX no domínio da matemática, a teoria da rela-

“judeu suíço”, não é menos verdade que se eu estiver um dia na situação de ser a "besta negra”, serei inversamente para os alemães um "judeu suíço" e para os ingleses um "cientista alemão" (Einstein, 1919a).

2 Ver Paty \& Freire e Wiener neste número. 
tividade geral no século xx levantou direta e concretamente, no domínio da física, ou seja, do mundo natural, estendido à consideração de todo o Universo, a questão da geometria que seria a mais adequada para a descrição desse mundo.

Geometria e experiência é o texto de uma conferência pronunciada em 27 de janeiro de 1921 na Academia de Ciências da Prússia, completado a seguir para a publicação nos Anais das Sessões (Sitzungberichte), nesse mesmo ano (Einstein, 1921b). Menos de dois anos antes, a observação astronômica (por ocasião do eclipse solar de maio de 1919, por uma dupla expedição à África e ao Brasil dirigida por Arthur Eddington para duas instituições britânicas, a Sociedade Real Científica e a Sociedade Astronômica) havia confirmado uma das grandes predições da teoria da relatividade geral de Einstein, obtida por este ao final do ano de 1915, a da curvatura dos raios luminosos que passam rasando o Sol ou, dito de outro modo, a curvatura do espaço na presença de grandes massas (cf. Dyson, Eddington \& Davidson, 1920). ${ }^{3}$ A observação ${ }^{4}$ parecia assim ter-se pronunciado sobre a natureza da geometria de nosso espaço, pelo menos na vizinhança de objetos massivos ou de campos gravitacionais intensos: ela era não eucliana e, nem mesmo, riemaniana (esférica). Mas, na verdade, as coisas não eram assim tão simples, a começar pela necessidade de definir exatamente não só o que se entende por "geometria", mas ainda de caracterizar a própria noção de "experiência" ou de "observação" e, como Einstein o esclarecerá bem, também a noção de "teoria física”, para a qual ele mostrará, de fato, sua centralidade nos juízos acerca desses assuntos.

A reflexão de Einstein, inseparavelmente matemática, física e filosófica, tratava precisamente dessa questão da natureza da geometria. Essa reflexão se postava com afastamento, sem qualquer triunfalismo, situando-se na seqüência dos debates que a haviam precedido, marcando entretanto uma mudança de apreciação relativamente a esses debates. $\mathrm{O}$ artigo de Einstein, que teve uma ampla difusão desde sua aparição e que foi traduzido em várias linguas, é publicado nesta edição em sua primeira tradução para o português. Retornaremos à análise de Einstein e ao contexto científico e filosófico que precedeu e acompanhou a redação desse texto que segue algum tempo após a elaboração de sua teoria da relatividade geral, freqüentemente qualificada também como "teoria geométrica da gravitação", bem como aos outros escritos de Einstein da mesma época que se ligam à questão da geometria.

3 Para apresentações e discussões das circunstâncias da observação: Mourão, 1987, 2003; Moreira \& Videira, 1995; Paty, 1996; Tolmasquin, 2005 .

4. Estritamente falando, tratava-se não de uma experiência, mas de uma observação, com medição precisa. Mas nessa ocorrência, a diferença entre experiência e observação, freqüentemente apontada particularmente a respeito da astronomia (onde não é possível intervir nas condições do objeto estudado), não é fundamental. 
O segundo texto referente à geometria, que o segue nesta edição, é muito menos conhecido e é apresentado em sua versão em espanhol, na qual foi publicado em 1926, após ter sido apresentado como conferência proferida em francês a partir de um manuscrito escrito em alemão, que infelizmente não pudemos encontrar (Einstein, 1926). 5 Intitulado La geometría no euclídea y la física, esse texto foi pouco divulgado, mas nem por isso deixa de ter um grande interesse e corresponde a uma abordagem original da parte de Einstein: insistindo sobre os aspectos epistemológicos e filosóficos da questão da geometria, tal como em Geometria e experiência, está situado quatro anos após este último. Ora, esses anos foram os da difusão no meio científico, e também para o grande público, das idéias da teoria da relatividade geral, como efeito da observação do eclipse solar de 1919 (sobre a curvatura do espaço) que tinha chamado a atenção do mundo inteiro sobre essa teoria. Esse período foi marcado por um florescimento intenso de publicações, não somente científicas e de vulgarização, mas também filosóficas, nas mais diversas direções (cf. Glick, 1987; Paty, 1979, 1985, 1987). Einstein se interessou vivamente por esses debates; não apenas porque era o autor da teoria científica que os ocasionava, mas por sua própria maneira de pensar, profundamente filosófica, que o conduzia a interrogar-se sobre a natureza do mundo e sobre o conhecimento (cf. Paty, 1993). Esboçaremos, também a propósito desse texto, uma análise de seu conteúdo bem como do contexto intelectual no qual ele se insere.

Einstein, de sua parte, perseguiu, durante toda sua vida, uma reflexão profunda sobre todos esses problemas, tanto científicos quanto filosóficos, intervindo, quando era solicitado, nos debates de sua época. Limitar-nos-emos aqui a tentar esclarecer a leitura dos dois textos, sem a pretensão de um estudo exaustivo. ${ }^{6}$

5 Este texto foi analisado, juntamente com outros escritos de Einstein sobre a geometria, em Paty, 1993, Cap. 7, p. 313- . Além do texto publicado originalmente em espanhol, reproduzido aqui, existe também uma tradução ao português por Antonio Augusto Passos Videira, revista por Mario Giambiaggi, publicada em Moreira \& Videira, 1995 . Einstein foi recebido como "membro honorário" da Academia Nacional de las Ciencias Exactas y Naturales (que se transformou na Sociedad Científica Argentina em 1929) em 16 de abril de 1625 e sua contribuição nesse dia foi a de responder a questões que tratavam essencialmente da física atômica e da radiação (cf. a transcrição em Academia, 1929). A conferência sobre La geometría no euclídea y la física foi pronunciada em outra ocasião. Sobre a física argentina nessa época, ver Westerkampf, 1975.

6 Pode-se ver um estudo mais detido e sistemático das concepções de Einstein sobre a natureza da geometria e sua relação com a física, postas em relação com os debates anteriores ou contemporâneos, em Paty, 1993, Caps. 6 e 7. 


\section{O DEBATE SOBRE A GEOMETRIA: DA DESGOBERTA DAS GEOMETRIAS NÃO-EUCLIDIANAS À TEORIA DA RELATIVIDADE GERAL}

A questão da geometria e de sua relação com o mundo físico foi explicitamente colocada desde a Antigüidade, desde a composição dos Elementos de geometria de Euclides e o enunciado do quinto postulado sobre as paralelas, que os geômetras se esforçaram por demonstrar durante dois mil anos (cf. Stäckel \& Engel, 1895; Houzel, 1990). Mas sua evidência aparente não era mais do que aquela, sensível, do mundo físico ao qual a geometria estava estreitamente ligada em sua origem (como Einstein o relembra em seus textos). Após o fracasso de todas as tentativas de demonstração do postulado das paralelas, qualificado por d'Alembert, no século xvııı, de "escândalo dos geômetras", consegue-se, pouco tempo depois, por meio das pesquisas de K. F. Gauss, J. Bolyai, N. I. Lobatchevski, B. Riemann, ${ }^{7}$ elaborar as condições de uma perspectiva muito diferente para a geometria, a saber, a multiplicidade de geometrias racionalmente possíveis, sem o quinto postulado, ou substituindo-o por outros axiomas, não sendo a geometria euclidiana mais do que uma delas. Desde então, a questão sobre a geometria modificou-se passando a ser a de saber qual dentre todas as geometrias possíveis a priori seria a mais apropriada para a descrição do espaço físico, ou as mais apropriadas, se a forma do espaço diferir de uma região para outra. Essa questão adquire uma maior precisão com a Dissertação de 1854 de Riemann intitulada Sobre as hipóteses que servem de fundamento para a geometria, mas que, apesar de sua profundidade, passou quase desapercebida em sua época, antes de fecundar todos os trabalhos posteriores sobre a geometria (Riemann, 1854).

De modo geral, a existência matemática de geometrias não-euclidianas inaugurou um debate científico e filosófico sobre a natureza da geometria apropriada ao mundo físico e sobre seu estatuto no conhecimento, e particularmente sobre a possibilidade de representar-se de maneira "intuitiva" um espaço correspondente a uma geometria não-euclidiana. A filosofia de Kant, que tinha privilegiado a geometria euclidiana a ponto de torná-la a referência da racionalidade pura enquanto “sintética a priori”, será seriamente posta em questão. Poder-se-ia invocar vários autores da segunda metade do século xix, protagonistas de debates sobre a natureza da geometria, tais como os matemáticos Pasch (1882) e Veronese (1891), que defendiam sobre bases diferentes posições empiristas, ou ainda B. Riemann, cujo racionalismo kantiano era temperado pelo realismo de Herbart e defensor do realismo do espaço (um espaço concebido fisi-

${ }_{7}$ Pesquisas preparadas principalmente pelos trabalhos de Saccheri, Lambert e Legendre. Cf. Freudenthal, 1957; Houzel, 1992. 
Introdução A três textos de Einstein...

camente e ligado indissociavelmente à matéria), W. K. Clifford (1876), E. Beltrami, H. Poincaré, ${ }^{\mathbf{8}}$ para as matemáticas, H. von Helmholtz $\left(1868,1882-1895,197^{8}\right)$ e E. Mach (1901, 1902, 1903, 1906), para a física e a psicofisiologia...

As duas posições mais marcantes do período podem ser remetidas àquelas de dois pensadores, dentre os cientistas-filósofos mais proeminentes, ambos marcados pelo pensamento kantiano mas em ruptura com esse pensamento, principalmente a propósito da filosofia da geometria. Uma vez admitida a origem pelo menos parcialmente empírica da geometria, uma vez constituída a geometria, ela é uma ciência racionalmente estruturada cuja relação com a natureza (com os corpos físicos) encontra-se de agora em diante posta em termos próprios. Helmholtz sustentava uma resposta empirista, considerando que as grandezas da geometria, em sua relação com o mundo físico, são providas de "relações de coordenação" que as relacionam a grandezas (coordenadas, distâncias) tomadas para os corpos físicos, o que define uma "geometria física" ou "geometria prática". A partir daí, a resposta é puramente experimental. A essa resposta empirista, Poincaré opunha a certeza racional que caracteriza as proposições da geometria enquanto ciência matemática à incerteza perpétua que as afetaria se contrariamente a geometria não fosse senão uma ciência experimental. Sua resposta consistia em que, dentre as diversas geometrias possíveis quando se considera sua relação com o mundo físico, sempre seremos livres para escolher por convenção aquela que conduzirá à solução mais simples.

Isso posto, é útil retornar por um instante à Dissertação de Riemann, na medida em que ela representa um papel considerável com relação àquilo que modificaria a natureza do debate sobre a geometria, a saber, a elaboração da teoria da relatividade geral. O estudo de Riemann estabelecia que, do ponto de vista puramente matemático, as variedades contínuas de um número qualquer de dimensões permanecem não-determinadas quanto a suas propriedades métricas. No caso particular das três dimensões, que correspondia, para Riemann, ao espaço concreto do mundo físico, são as propriedades dos corpos físicos contidos no espaço que determinam as propriedades métricas, ou dito de outro modo, a estrutura do espaço (seu caráter euclidiano ou "plano" ou, ao contrário, não-euclidiano e curvo). Riemann sublinhava principalmente que a questão de saber como o espaço é nos domínios que escapam a nossa apreensão perceptual e a nossa experiência, aqueles domínios das dimensões "incomensuravelmente pequenas e incomensuravelmente grandes", era uma questão inteiramente aberta (Riemann, 1854).

8 Poincaré, 1895, 1898, 1902a, 1902b, 1903, 1907, 1908, 1912a 1912b. Sobre Poincaré, ver Toretti (1978); Vuillemin (1972). 
Após essa primeira renovação da questão da natureza da geometria ocasionada pelas geometrias não-euclidianas, a teoria da relatividade geral suscitará uma segunda renovação. Com efeito, esta última teoria apresentar-se-á, desde sua concepção, como uma teoria física, que permitia pôr de maneira efetiva e precisa a questão da implicação das geometrias não-euclidianas na concepção do mundo físico, uma vez que ela comportava em sua própria construção elementos dessas geometrias; ela permitia que se desse de um modo ou de outro uma resposta, formulando assim a estrutura métrica do espaço físico. 9

De certo modo, sessenta anos após o anúncio da maneira de ver de Riemann, a teoria da relatividade geral vinha responder à questão levantada por este último. Ou, antes que uma resposta no sentido estrito, seria mais justo falar de uma correspondência entre as duas: a teoria física da relatividade geral, ou teoria relativista (geral) da gravitação, fazia eco à demanda de Riemann formulada a partir de considerações matemáticas, tanto mais que ela havia encontrado na geometria riemanniana o instrumento matemático que melhor permitia formular o problema posto pela física. A construção da teoria física determina inegavelmente uma nova perspectiva sobre a relação da geometria com a física.

O debate sobre a natureza da geometria do mundo físico e sobre sua decidibilidade experimental não podia, portanto, após a teoria da relatividade geral, simplesmente reproduzir as considerações epistemológicas anteriores, ainda que se nutrisse delas. A posição de Einstein retira, como veremos, toda sua originalidade do fato de ter percebido plenamente isso face às considerações dos empiristas lógicos que se contentarão em simplesmente retomar os termos anteriores do problema (cf. Einstein, 1921b, 1926, 1949). Pois, em todos os casos, é à teoria da relatividade geral que vai a referência da questão da escolha entre as geometrias. Em outros termos, com relação às outras teorias da física, a relatividade geral é aquela que torna necessário um pensamento das relações entre a geometria e a física e a incorporação da primeira pela segunda, além de permitir a extração - no sentido de Riemann - de todas as conseqüências, na medida em que é sobre esse pensamento que ela funda sua própria possibilidade.

Einstein abordará explicitamente o problema da geometria em sua relação com a física pela primeira vez, em 1916, em seu artigo fundador da teoria da relatividade geral (Einstein, 1916), artigo esse que se fundamenta sobre os resultados obtidos por ele ao final do ano anterior (Einstein, $1915^{\mathrm{a}}, 1915^{\mathrm{b}}, 1915^{\mathrm{c}}$ ). Ela já o tinha, de fato, abordado "praticamente" em sua elaboração da teoria da relatividade restrita, preparando então sem o saber o caminho para a teoria da relatividade geral (Einstein, 1905, 1907,

9 Einstein, 1916, 1917a, 1921a; Einstein et al., 1922; Weil, 1922 [1918]; Howard \& Stachel, 1989; Toretti, 1983; Paty, 1992, 1993, 1995 . 
1987[1912]; cf. Paty, 1993, Caps. 2 a 5). Note-se que a geometria do espaço é aqui estendida àquela do espaço-tempo. A geometria do espaço-tempo da teoria da relatividade restrita era uma geometria (temporal) física ou prática no sentido de Helmhotz, e suas grandezas comportavam um conteúdo físico correspondente ao dos corpos rígidos euclidianos e ao das durações uniformes.

A relatividade geral, sublinha Einstein nesse trabalho, "não pode aderir a essa interpretação física simples do espaço e do tempo" (Einstein, 1916, parte A, parágrafo 1, grifo meu). A extensão do postulado de relatividade (restrita aos movimentos inerciais) em princípio da relatividade generalizada, segundo o qual "as leis da física se aplicam do mesmo modo aos sistemas de referência em qualquer movimento", implica considerações sobre a forma espacial dos corpos de referência que constituem esses sistemas, que devem passar pelo abandono dessa interpretação (Einstein, 1916, parte A, parágrafo 2). Com efeito, a idéia da "geometria física" ou "prática" (formada a partir daquela de "corpos de referência") e sua crítica constituirá, de certo modo, a matriz da elaboração da teoria da relatividade geral de Einstein. Ao abandonar a interpretação física simples da geometria-tempo da mecânica clássica e da relatividade restrita - o espaço-tempo de Minkowski (1915 [1907], 1909) -, Einstein retornava ao espaço(-tempo) sem interpretação física da variedade matemática riemanniana, ou seja, sem forma (sem métrica definida), e é sobre esse "molusco de referência" que ele podia raciocinar matematicamente, sem o obstáculo da limitação (quase-)euclidiana. Ao final da elaboração formal guiada pela escolha dos princípios, transcritos em condições de relações sobre essas grandezas, as novas relações estabelecidas forneciam o conteúdo da nova métrica. Tal foi a resposta efetiva da teoria física da relatividade geral de Einstein à consideração geométrica de Riemann.

Todo o trabalho que, no artigo de 1916, segue essas considerações iniciais, consiste, após uma preparação do instrumento matemático apropriado (o cálculo tensorial), na construção de uma teoria (do campo gravitacional) fundada sobre a noção (posta como exigência física e formal) de covariância geral. Ultrapassou-se, de certo modo, o problema da interpretação dos conceitos de espaço e de tempo, que constituiu o momento crítico preliminar, pela construção de uma teoria física. Um pouco mais tarde, em 1917, Einstein estendeu essa consideração à constituição de uma teoria do universo físico em seu conjunto (Einstein, 1917b), o que deu um outro desenvolvimento à questão da geometria, desde então abordado por Einstein em suas reflexões sobre esse tema.

Essas reflexões são retomadas ou empreendidas em outros escritos - científicos e de vulgarização ou epistemológicos e filosóficos: os primeiros contêm sempre elementos epistemológicos e, nos segundos, a teoria física está presente, pois é ela que fornece uma doutrina do espaço - tais como o livro de 1917 dirigido ao grande público, $A$ teoria da relatividade restrita e geral, as Conferências na Universidade de Princeton de 1921, 
um artigo publicado em 1920 sobre o éter gravitacional como espaço físico e, finalmente, o artigo Geometria e experiência de 1921 (Einstein, 1917a, 1920, 1921a, 1921b). Nesses textos, a relação da geometria à física é vista agora como o fio condutor subjacente ao percurso de conjunto que conduz da mecânica clássica à relatividade restrita e até a relatividade geral, ao mesmo tempo que lança luz sobre a evolução do pensamento de Einstein em direção a uma explicitação do problema dessa relação, ocasionada pela teoria física que ele acaba de desenvolver.

Em todos esses escritos (assim como naqueles que seguirão), a noção física invocada de corpos de referência permite a Einstein situar-se com relação ao empirismo de Helmholtz e com relação ao convencionalismo de Poincaré. Do primeiro, ele empresta a constituição física de um espaço (estendido ao espaço-tempo), e compartilha com o segundo a consciência da racionalidade da geometria e de uma certa liberdade de escolha quando se leva a imbricação da geometria e da física na consideração de situações concretas. Todavia, como explica Einstein, o físico não teria nada a fazer com a geometria se ele não se preocupasse em "coordenar" suas noções fundamentais com os "objetos reais". Ele mostra, por meio dessa coordenação, que "há um sentido em falar da verdade ou da exatidão das proposições geométricas". A "geometria euclidiana assiminterpreta$d a$ " (Einstein, 1921a, grifo meu) exprime algo diferente do que proposições que resultariam unicamente das definições dessas noções sem referência aos corpos (Einstein, 1921a). É nisso que a posição de Einstein mais se distingue da posição de Poincaré.

\section{O ARTIGo de 1921}

Em Geometria e experiência (Einstein, 1921b), que é um dos artigos mais importantes do ponto de vista estritamente epistemológico, Einstein se detém inicialmente na questão da natureza da matemática, ou seja, seu caráter abstrato, axiomático, e sua capacidade de aplicar-se às ciências da natureza. Ele inicia sua reflexão a respeito da matemática, debruçando-se sobre as concepções da axiomática moderna e, relativamente à geometria, sobre a geometria axiomática, as quais permitiram esclarecer a relação da matemática com a realidade (cf. Russell, 1897; Hilbert, 1899), fazendo uma distinção nítida, nas proposições da matemática entre a parte lógico-formal e o conteúdo objetivo ou intuitivo, que anteriormente se confundiam: somente a primeira constitui o objeto próprio da matemática. É nesse espírito que ele anuncia sua afirmação desde então célebre:

Na medida em que as proposições das matemáticas se relacionam com a realidade, elas não são certas, e na medida em que são certas, elas não se relacionam com a realidade. 
Introdução a três textos de Einstein...

Essa concepção da axiomática moderna "libera a matemática de todos os elementos que lhe são estranhos e dissipa assim a obscuridade mística que envolvia anteriormente seus fundamentos", de sorte que a matemática está separada do mundo real. ${ }^{10}$ A possibilidade de um acordo entre eles é obtido pela constituição de uma geometria prática, cujo objeto é o estudo dos corpos idealizados (na medida em que são relacionados a suas propriedades geométricas), relacionados a corpos reais "acessíveis à experiência", e que é assim uma ciência da natureza, de fato, "o ramo mais antigo da física" (Einstein, 1921b). ${ }^{11}$

Segue-se, então, que o problema da decisão acerca das proposições dessa ciência pelo recurso à experiência não é diferente do recurso à experiência da própria física. A conclusão opõe-se à de Poincaré, apesar da proximidade das posições de partida:

A questão de saber se a geometria prática do mundo é euclidiana ou não tem um sentido preciso e a resposta não pode ser fornecida a não ser pela experiência (Einstein, 1921b).

Poincaré acantonava a geometria nas definições e considerava que as propriedades físicas são dissociadas dessas definições, enquanto que Einstein institui a "geometria prática" como instância intermediária, ao mesmo tempo física e definitória. Esta última o conduz, a seguir, à formulação de uma teoria física mais elaborada (a teoria da relatividade). A separação que fazia Poincaré, entre a geometria que depende do entendimento e os corpos físicos que se relacionam com a experiência sensível, é assim ultrapassada por uma concepção da teoria física que se liga tanto ao intelecto como à experiência sensível.

Em sua discussão das idéias de Poincaré (que ele retomará freqüentemente a propósito da geometria), Einstein atribui a esta última uma concepção da "indissociabilidade" entre a geometria e a física que não é exatamente a posição de Poincaré (ela foilhe atribuída por Moritz Schlick), mas que Einstein retoma por conta própria. Não é somente a geometria que fornece os enunciados sobre o comportamento dos objetos reais, mas a geometria $(G)$ combinada ao conjunto das leis físicas $(P)$ : "é somente a soma $(G+P)$ que está submetida ao controle da experiência"; e pode-se, em princípio, fazer uma escolha convencional. "Sub specie aeterni a concepção de Poincaré parece-me correta", indica ele, sublinhando que, com efeito, não há no mundo real objetos que

10 Einstein faz referência ao livro de Schlick sobre a teoria do conhecimento; cf. Schlick, 1918.

11 O termo "coordenar" será retomado por Reichenbach para falar das “definições de coordenação". Infelizmente não podemos analisar aqui as concepções de Reichenbach e sua discussão por Einstein (cf. Reichenbach, 1920, 1922, 1978; Einstein, 1949; Paty, 1993, Cap. 7). 
correspondem exatamente aos objetos-padrões ideais da geometria (Einstein, 1921b). ${ }^{\mathbf{1 2}}$ De fato, Poincaré situava a geometria nas definições, que podem ser mudadas como se muda na física as unidades escolhidas. Não é, portanto, exatamente o ponto de vista de Poincaré que é assim descrito, mas sobretudo uma tradução desse ponto de vista feita por Einstein em sua própria perspectiva, ou seja, em termos de geometria física ou prática. Na descrição que apresenta do problema é, em realidade, (Gpr $x P$ ) que se tem e não $(G+P)$, Gpr designando a geometria prática no sentido indicado acima.

Einstein leva a indissociabilidade por princípio ainda mais longe do que aquela que ele atribui a Poincaré, fazendo observar que os objetos-padrões, que são o corpo sólido e o relógio, "não possuem o papel de elementos irredutíveis no edifício conceitual da física, mas somente aquele de estruturas compostas, cujo conjunto por elas constituído não deve ser independente da física teórica". O conhecimento delas remete ao horizonte da futura teoria unificada da matéria, que incluiria suas propriedades quânticas. Ele retomará mais tarde esse ponto de vista holista sob uma forma semelhante, mas ainda mais precisa (no texto de 1949), contra a concepção empirista da geometria. Deve-se, portanto, na prática, em razâo dessa limitação de nosso conhecimento, ultrapassar a indissociabilidade por princípio e contentar-se com uma aproximação razoável, na qual uma escolha será sempre necessária. A objeção de convencionalismo estrito é assim afastada em nome de uma atitude prática, de um conhecimento aproximado. Essa refutação, que os empiristas lógicos retomarão por sua vez, Einstein não a concebe, ao contrário destes últimos, como um argumento empirista contra o convencionalismo. O emprego do termo "realidade física", e não do termo "experiência" (que será utilizado pelos empiristas), é aqui repleto de significação: é num sentido realista, e não empirista, que Einstein propõe ultrapassar (e não negar) o convencionalismo, e sua posição não é simplesmente pragmática. Ela está subtendida em uma concepção da construção da teoria física.

Dois aspectos importantes da epistemologia de Einstein estão presentes nessa argumentação: de um lado, os conceitos da geometria pura, da geometria prática, ou da física são construídos pelo pensamento e são condicionados por princípios apropriados aos objetos a serem descritos e ao objetivo da descrição; de outro lado, os conceitos da física são constituídos matematicamente como elementos de representação do mundo físico.

12 Ver também Einstein (1929):

Ainda que os constituintes estruturais da matéria (tais como o átomo e o elétron, por exemplo) supostos pela física não sejam em princípio comensuráveis com os corpos rígidos, ainda assim aplicamo-lhes os conceitos da geometria [...] Por essa razão, pensadores consistentes inclinaram-se para a não permissão de fazer corresponder os conteúdos fatuais reais (reale Tatsachenbestände) unicamente à geometria. Estimaram ser preferível fazer corresponder os conteúdos da experiência (Erfahrungbestände) à geometria e à física conjuntamente. 
Einstein aborda a seguir, em Geometria e experiência, a questão da interpretação física da geometria a propósito das duas regiões limites do mundo físico e retoma o exame das razões para adotar a hipótese de um universo finito, na direção do "princípio de Mach", retomado e precisado pela própria teoria da relatividade geral. Para desfazer-se da ação-à-distância inerente ao princípio de Mach, deve-se modificar esse princípio, fazendo a hipótese de um "éter" (ou seja, de fato, de um espaço físico) que transmite os efeitos da inércia, ou seja, aquele da relatividade geral. Esse "éter", do qual Einstein fala em outro texto da mesma época, e que ele chama de "éter de Mach", "não determina somente o estado das massas inertes, mas é ele próprio determinado por elas" (Einstein, 1920).

Einstein põe então a questão da representação intuitiva de um universo finito de três dimensões e, no entanto, sem limites: essa questão, de agora em diante, não é mais somente a questão da intuição que podemos ter de uma geometria para um espaço abstrato, pois ela se vê carregada, com a teoria da relatividade e a cosmologia, de todo o peso da representação do próprio mundo físico real. Para Einstein, uma tal representação é possível, contrariamente à opinião mais difundida. $\mathrm{O}$ alcance da "representação intuitiva" vai, aos seus olhos, bem além da simples crítica da concepção kantiana estrita do espaço e da geometria, crítica na qual o foco é, ao contrário, a argumentação dos filósofos neoempiristas e neopositivistas, Carnap e Reichenbach (cf. Paty, 1993, Cap. 7), para tocar a própria natureza daquilo que se entende por teoria (física), em sua lógica e na sua relação possível com a experiência, isto é, à função da teoria para nosso entendimento; e é isso que o retém em sua análise. Aqui, remetemos ao próprio texto de Einstein, o qual, ao propor mais que uma imagem, uma verdadeira experiência de pensamento, que põe em jogo os próprios conceitos que caracterizam o problema, conclui pela possibilidade de uma "representação intuitiva da geometria esférica, apoiando-se na maneira de pensar e de visualizar oferecidas pela geometria euclidiana. (Esta questão já havia sido abordada por Riemann, Helmholtz, Mach, Poincaré e outros.)

Note-se que essa representação visualizada é obtida por uma reconstrução racional de nossa intuição, que substituiu as imagens comuns por jogos de relações. $\mathrm{Na}$ medida em que essas relações tornam-se visuais por apelo à experiência mental, pode-se dizer que são intuitivas (Einstein, 1921b). ${ }^{13}$ 


\section{O DEBATE FILOSÓFICO DOS ANOS 1920}

\section{E O ARTigo de 1926 SOBRe O PONTO DE VISTA DE RiemanN}

$\mathrm{O}$ artigo de Einstein que acabamos de analisar constituirá uma referência importante nas discussões sobre o problema da geometria tal como ele se viu renovado pela teoria da relatividade geral. Ele tem seu lugar, juntamente com os outros textos de Einstein já mencionados, na abertura do debate científico e filosófico, cujos elementos essenciais e as posições em concorrência se encontram dispostas desde o início dos anos 1920. O próprio Einstein foi muito informado dos argumentos de uns e de outros e essas diversas contribuições servem de pano de fundo a suas próprias observações (e principalmente àquela de sua intervenção culminante de 1949 que o oporá a Reichenbach e na qual se apoiará, pelo menos no início, em Poincaré, em um diálogo imaginário entre o empirismo "verificacionista" de Reichenbach e o "não-positivismo" da concepção realista crítica que é de fato a sua posição). ${ }^{\mathbf{1 4}}$

O debate sobre a questão da geometria tinha, na verdade, sido deflagrado desde a publicação da teoria de Einstein, e os primeiros textos que então a invocaram foram as obras de vulgarização que começaram a aparecer desde 1917; em particular, aquela já mencionada de Einstein, A teoria da relatividade restrita e geral, e aquela de Moritz Schlick, O espaço e o tempo na física contemporânea, que tinha como subtítulo: Uma introdução à teoria da relatividade e da gravitação (Einstein, 1917̧a; Schlick, 1917). Os dois apresentam então um ponto de vista bastante próximo, na direção que será a seguir aprofundada por Einstein. Schlick intervirá posteriormente em outros textos defendendo uma posição cada vez mais empirista, e propondo, tal como Reichenbach fazia de seu lado, a "dissolução do sintético a priori", posição que acompanhará a formação do positivismo ou empirismo lógico juntamente com a "concepção verificacionista do significado" (Schlick, 1915, 1916, 1917, 1918, 1921a, 1921b; Reichebach, 1920, 1924, 1928, 1949). Rudolf Carnap publicará igualmente a partir dos anos 1920 seus primeiros textos que tratam da geometria. $\mathrm{O}$ criticismo kantiano e o neo-kantismo foram os alvos privilegiados desses estudos (Carnap, 1921, 1924, 1925). Ernst Cassirer, neo-kantiano e membro eminente da Escola de Marburg, encontrará, ao contrário, na teoria da relatividade, restrita e geral, um motivo perfeitamente apropriado para desenvolver a respeito dela as idéias propostas anteriormente em Substância e função, livro publicado por ele em 1621. A teoria da relatividade de Einstein foi rapidamente objeto de uma tradução para o inglês, na qual os dois textos foram conjuntamente publicados em um só volume; o que testemunha a favor da atualidade de seu propósito e da vitalidade de seu ponto de vista neo-criticista, por meio do qual ele refundava certas noções kantia-

14. Einstein, 1949, escrito em resposta a Reichenbach, 1949; cf. Paty, 1993, Cap. 7, p. 336-7. 
Introdução A três textos de Einstein...

nas, propondo em conseqüência uma visão interessante sobre o espaço e a geometria (ele punha na origem dessas noções a "função de espacialidade", que evitava a dificuldade de um sintético a priori rígido (cf. Cassirer, 1910, 1921, 1923). O debate assim iniciado entre o apriorismo, um criticismo reformado, o empirismo, o convencionalismo e o realismo (crítico) perdurou ao longo dos anos.

É nesse contexto que se localiza o texto de Einstein publicado em 1926, La geometría no-euclídea y la física, que trata essencialmente da origem e da natureza da geometria e da importância do ponto de vista de Riemann.

O interesse desse texto de Einstein, em relação a suas outras contribuições sobre o assunto, é que ele, nesse texto, coloca-se do ponto de vista da geometria, mais do que do ponto de vista da física, visando sua origem, sua história (principalmente o desenvolvimento das geometrias não-euclidianas) e sua natureza. Mas essa outra maneira de abordar a questão da geometria - mais conforme àquela dos matemáticos - não conduz a conclusões diferentes daquelas que tinham imediata e mais diretamente como eixo a teoria da relatividade geral. Além disso, não é por acaso que Einstein é conduzido, mesmo nessa via, na conclusão de sua exposição, a sublinhar a importância do ponto de vista de Riemann.

A exposição parte da geometria considerada em si mesma para chegar à questão de sua natureza, a sua junção com a relatividade geral. Considerada da perspectiva de sua origem e de sua história, a geometria transformou-se do estado de conhecimento ligado à experiência àquele de uma ciência matemática. Um primeiro esclarecimento é aqui interessante, a propósito da origem empírica da geometria, já abordado precedentemente (caráter empírico esse sobre o qual tinham insistido Helmholtz, Mach e Poincaré). Einstein toma o cuidado de indicar que essa origem não é unicamente empírica, e que, enquanto ciência, a geometria não era, em sua origem, somente experiência, uma vez que a experiência já era mediatizada por uma teoria. Quanto à transformação da geometria em uma ciência matemática, Einstein a religa às condições da constituição das próprias matemáticas, como sistemas lógicos e formais independentes das contingências da experiência.

Mas, após esse processo de abstração, acaba-se por esquecer a origem empírica dos conceitos e dos axiomas da geometria, e por considerar que "lhe correspondem os objetos da intuição interna", de tal sorte que negar um axioma da geometria seria contrário ao bom senso. Essa crítica dos conceitos ou das proposições rígidas, fixadas em sua formulação abstrata, uma vez esquecidas as condições de sua formação, lembra as críticas de Mach, de quem Einstein sempre se disse tributário acerca desse ponto (ver, por exemplo, Einstein, 1929). Apresentado sob essa forma, o problema da possibilidade de adaptar os axiomas assim fundados aos objetos da realidade implica a concepção kantiana do espaço. 
A utilização da geometria é necessária para exprimir as leis da física (o argumento é retomado de Helmholtz, mas sua conseqüência é aqui transformada), de onde se segue que a geometria vem antes da física, e que ela "aparece como precedendo logicamente toda a experiência e toda a ciência experimental". Daí a idéia, nos matemáticos, nos filósofos e nos físicos do início do século xIx, de que os fundamentos da geometria eram intangíveis. Mas Einstein relembra também que é nas matemáticas puras que se realizou a superação da geometria euclidiana. Assim, mesmo na crítica dos fundamentos da geometria, Einstein faz ver que a geometria precede ainda as outras ciências, pelo menos até o dia em que se percebeu que "a vestimenta da geometria euclidiana [era] muito estreita para a física".

Ele passa a evocar as discussões sobre o fato de saber se essa última questão tem um sentido, discussões que remetem no essencial às posições respectivas de Helmholtz e de Poincaré, que Einstein descreve respectivamente como o ponto de vista da (geometria) "física prática"15 (e não, cabe ressaltar, aquele do empirismo), e como aquele do convencionalismo. O primeiro foi indispensável para desenvolver a teoria da relatividade geral. Mas pode-se também considerar, de uma outra perspectiva, que não existe objeto correspondente aos conceitos fundamentais da geometria, e que, nesse sentido, "a geometria isolada não contém enunciado algum sobre os objetos reais". Einstein retoma a diferença entre a geometria pura e axiomática e a "geometria prática", como em Geometria e experiência. Com a segunda, a questão da validade ou não da geometria euclidiana tem um sentido claro. A geometria "se transforma em uma ciência física quando os axiomas contêm afirmações sobre os objetos naturais, afirmações das quais somente a experiência pode decidir a exatidão".

Einstein evoca ainda, a esse propósito, os dois domínios-limite do cosmo e dos átomos e as considerações propostas por Riemann, a quem ele consagra, de maneira significativa, o fim de sua exposição. A geometria riemaniana é a mais geral e engloba a geometria euclidiana bem como as várias geometrias não-euclidianas a título de casos particulares. Riemann deu-se conta de que os axiomas da geometria não são suficientes para determinar a métrica. Chegou assim, pela pura especulação matemática, ao pensamento da "indissociabilidade da geometria e da física". É, portanto, a Riemann que Einstein remete, em última análise, essa indissociabilidade, à qual a teoria da relatividade geral dará, sessenta anos mais tarde, realidade, no sentido de que "geometria e teoria da gravitação se fundem um uma única entidade" (Einstein, 1926).

15 O texto da transcrição em espanhol indica "física prática”. É tentador substitui-lo por "geometria prática", em conformidade com a expressão utilizada em Geometria e experiência, tanto mais que o sentido no qual é empregada é de fato o mesmo. Trata-se, como vimos, da "geometria física" ou "prática". Talvez o termo "geometria prática" tenha parecido estranho a quem transcreveu o texto, que poderia tê-lo substituído por "fisica prática”. É isso o que eu supus, ao restabelecer "geometria prática" nas duas citações que seguem. 
Introdução A três textos de Einstein...

Temos, na verdade, com essa afirmação, a essência da concepção de Einstein sobre a decidibilidade da geometria: ela é a mesma da teoria física que dá o conteúdo (métrico) da geometria.

Em resumo, esse texto de Einstein confirma e completa o que resultava de suas análises anteriores, mostrando explicitamente como se pode concluir pela possibilidade de decidir a geometria pela experiência, afirmando, ainda assim, a indissociabilidade da geometria e da física. O ponto de vista da geometria prática ou física (de Helmholtz) não constitui um ponto de chegada conclusivo, mas um ponto de passagem na direção da construção de uma teoria física (geometrizada) que tinha sido posta como uma necessidade por Riemann. A teoria da relatividade geral aparece, a partir de então, como essa teoria física que modifica de modo decisivo os termos do problema sobre a natureza da geometria e permite ultrapassar a oposição de Helmholtz e de Poincaré, do empirismo e do convencionalismo.

\section{Conclusão}

Após os textos que acabamos de analisar e que são publicados a seguir, Einstein não retornará essencialmente ao problema das relações da geometria e da física que muitos anos mais tarde, para marcar sua divergência de visão com a concepção empirista, da qual Reichenbach era um dos principais representantes. Uma passagem importante de sua Resposta às críticas de 1949, na obra de P. A. Schilpp em sua homenagem, é consagrada a essa questão e constitui - quanto ao pensamento de Einstein - de certa maneira o auge da discussão que preocupou os filósofos durante mais de dois decênios após a elaboração da relatividade geral. Einstein já tinha apresentado o essencial de suas reflexões sobre a questão da geometria em sua relação com o mundo físico. Ele retornaria a isso novamente em outras contribuições, principalmente em $A$ relatividade e o problema do espaço de 1954 (Einstein, 1954,b), para esclarecer ainda, se isso fosse possível, seu pensamento sobre o assunto, no qual a teoria da relatividade geral sempre em devir perseguia seu diálogo com o pensamento da geometria segundo Riemann.

As questões levantadas pela relação da geometria com o mundo físico permanecem atuais, e o debate sobre esse tema está ainda vivo, ligado a outras questões fundamentais sobre o conhecimento, e alimentado principalmente pelos novos conhecimentos adquiridos nas duas regiões extremas já indicadas por Riemann - às quais Einstein se refere bem particularmente no texto de 1926 -, a saber, as regiões do microcosmo e do macrocosmo, exploradas respectivamente pela física quântica e pela cosmologia. 


\section{REFERÊNGIAS BIBLIOGRÁFIGAS}

Academia Nacional de Ciencias Exactas, Físicas y Naturales de Buenos Aires: recepción del doctor Alberto Einstein en la sesión especial de la Academia el dia 16 de abril de 1925. Anales de la Sociedad Científica Argentina, 107, p. 337-47, 1929.

Boi, L.; Flament, D. \& Salanski, J. M. (Ed.). 1830-1930: un siècle de géométrie, de C. F. Gauss et B. Riemann à H. Poincaré et E. Cartan. Epistémologie, histoire et mathématiques. Berlin: Springer-Verlag, 1992.

Carnap, R. Der Raum. Ein Beitrag zur Wissenschaftlehre. Iena: 1921.

. Dreidimensionalität des Raumes und Kausalität. Annalen der Philosophie, 4, p. 105-3o, 1924.

. Ueber die Abhängigkeit der Eigenschaften des Raumes von denen der Zeit. Kantstudien, 3o, p. 33145,1925 .

Cassirer, E. Substanzbegriff und Funktionsbegriff. Berlin: Bruno Cassirer, 1910. (Trad. inglesa: Substance and function. In: Cassirer, E. Substance and function and Einstein's theory of relativity. Chicago: Open Court, 1923. p. 1-34,6.)

.Zur Einstein'schen Relativitätstheory. Berlin: Bruno Cassirer, 1921. (Trad. inglesa: Einstein's theory of relativity considered from the epistemological standpoint. In: CAssirer, E. Substance and function and Einstein's theory of relativity. Chicago: Open Court, 1923. p. 347-460.)

. Substance and function and Einstein's theory of relativity. Trad. de W. C. Swabey \& M. C. Swabey. Chicago: Open Court, 1923.

Clifford, W. K. On the space-theory of matter. Proceedings of the Cambridge Philosophical Society, 2, p. $157^{-8}, 1876[1870]$.

Dyson, F. W.; Eddington, A. S. \& Davidson, G. A determination of the deflection of light by the Sun's gravitational field from observations made at the total eclipse of may 29, 1919. Philosophical Transactions of the Royal Society, p. 291-333, 1920.

Einstein, A. Elektrodynamik bewegter Körper. Annalen der Physik, ser. 4, 17, p. 891-921, 1905. (In: Stachel, J.; Cassidy, D. et al. (Ed.). The collected papers of Albert Einstein. Princeton: Princeton University Press, 1989. v. 2, p. 275-306. Trad. francesa: Einstein, A. Oeuvres choisies. Paris: Seuil/CNRS, 19891993. v. 2, p. 31-58.)

. Ueber das Relativitätsprinzip und die aus demselben gezogenen Folgerungen. Jahrbuch der Radioaktivität und Elektronik, 4, p. 411-62, 1907. (Trad. inglesa: Stachel, J.; Cassidy, D. et al. (Ed.). The collected papers of Albert Einstein. Princeton: Princeton University Press, 1989. v. 2, p. 433-84. Trad. francesa: Einstein, A. Oeuvres choisies. Paris: Seuil/CNRS, 1989-1983.v. 2, p. 84-124.)

. Ueber das Relativitätsprinzip und die aus demselben gezogenen Folgerungen. Jahrbuch der Radioaktivität und Elektronik (Berichtigungen), 5, p. 98-9, 1908. (Trad. inglesa: Stachel, J.; CAssidy, D. et al. (Ed.). The collected papers of Albert Einstein. Princeton: Princeton University Press, 1989. v. 2, p. $494-5$.) 
Introdução a três textos de Einstein...

Antrittsrede. Preussische Akademie der Wissenschaften, Sitzungsberichte, part 2, p. 739-42, 1914. (Discours de réception à l'Académie des sciences de Prusse. In: . Oeuvres choisies. Paris: Seuil/

CNRS, 1989-1993.v. 5, p. 92-4.)

Die Relativitätstheorie. Die Physik, Leipzig, 3, 1, p. 7o3-16, 1915a.

. Zur allgemeinen Relativitätstheorie. Preussische Akademie der Wissenschaften, Sitzungsberichte, part.

2, p. 778-86, p. 799-8o1, 1915b.

Die Feldgleichungen der Gravitation. Preussische Akademie der Wissenschaften, Sitzungsberichte, part 2, p. $844-47,1915^{\mathrm{c}}$.

. Die Grundlage der allgemeinen Relativitätstheorie. Annalen der Physik, ser. 4, 4, 9, p. 769-822, 1916. (Les fondements de la théorie de la relativité générale. In: Oeuvres choisies. Paris: Seuil/CNRS, 1989-1993.v. 2, p. 179-227.)

. Ueber die spezielle und die allgemeine Relativitätstheorie, Gemeinverständlich. Vieweg: Braunschveig, 1917̨a. (La théorie de la relativité restreinte et générale. Trad. de M. Solovine. Paris: Gauthier-Villars, 1954.)

Kosmologische Betrachtungen zur allgemeinen Relativitätstheorie. Preussische Akademie der Wissenschaften, Sitzungsberichte, part 1, p. 142-52, 1917b. (Considérations cosmologiques sur la théorie de la relativité générale. In: Oeuvres choisies. Paris: Seuil/CNRS, 1989-1993.v. 3, p. 88-98.)

My theory. The Times. London, 28 Nov. 1919a, p. 13.

Induktion und Deduktion im Physik. Berliner Tageblatt, 21 Dez. 1919b. (Induction et déduction en physique. In: . Oeuvres choisies. Paris: Seuil/CNRS, 1989-1993. v. 5, p. 94-6.)

.Aether und Relativitâtstheorie: Rede Gehalten am 5 . Mai 1920 an der Reichs-Universitât zu Leiden. Berlin: Springer, 1920. (L'éther et la théorie de la relativité. In: Oeuvres choisies. Paris: Seuil/CNRS, 19891993.v. 5, p. 81-8.)

.Vier Vorlessungen ûber Relativitätstheorie. Vieweg: Braunschveig, 1921a. (The meaning of relativity: four lectures delivered at Princeton University. Trad. de E. P. Adams. Princeton: Princeton University Press, 1922.)

. Geometrie und Erfahrung. Preussische Akademie der Wissenschaften, Sitzungsberichte, part 1, p. 1233०, 1921b. (La géométrie et l'expérience. In: . Oeuvres choisies. Paris: Seuil/CNRS, 1989-1993. v. 5 , p. 70-81.)

. Geometría no euclídea y física, Revista Mathematica Hispano-Americana, ser. 2, p. 72-6, 1926.

Space-time. Encyclopedia Britannica, 1929. v. 21, p. 105-8.

Reply to criticism. Remarks concerning the essays brought together in this cooperative volume. In:

Schilpp, P. A. (Ed.). Albert Einstein: philosopher-scientist. La Salle: Open Court, 1949. v. 2, p. 663-93. . Ueber die Spezielle un die Allgemeine Relativitâtstheorie. Vieweg: Braunschveig, 1954a.

. Relativität und Raumproblem. In: . Ueber die Spezielle un die Allgemeine Relativitâtstheorie. Vieweg:

Braunschveig, 1954b. p. 151-76.

. Einstein's manuscript on the special theory of relativity. In: KLEIn, M.; Kox, A. J. et al. (Ed.). The collected papers of Albert Einstein. Princeton: Princeton University Press, 1995 [1912]. v. 4, p. 9-108. . Oeuvres choisies. Paris: Seuil/CNRS, 1989-1993. 5 v.

Einstein, A.; Lorentz, H. A.; Minkowski, H. \& Weyl, H. Das Relativitätsprinzip. 4 ème éd. Leipzig: Teubner, 1922. (The principle of relativity. Trad. de W. Perrett \& G. B. Jeffery. London: Methuen, 1923.)

Évora, F. R. (Ed.). Espaço e tempo. Campinas: Unicamp / CLE, 1995.

Freundenthal, H. Zur Geschichte der Gründlagen der Geometrie. Niew Archief voor Viskunde, 4, p. $105^{-}$ 42,1957 .

GLick, T. (Ed.). The comparative reception of relativity. Dordrecht: Reidel, 1987. 
Heцmнoltz, H. L. F. von. Ueber die Tatsachen, die der Geometrie zugrunde liegen. Nachrichten von der königlichen Gessellschaft der Wissenschaften zu Göttingen, 9, 1868. (On the facts underlying geometry. In:___. Epistemological writings. Introd. e bibliografia de R. S. Cohen \&Y. Elkana. Dordrecht/Boston: Reidel, 1978. p. 39-71.

Wissenschaftliche Abhandlungen. Leipzig: [s.n.], 1882-1895. 3 v.

. Epistemological writings. Introd. e bibliografia de R. S. Cohen \& Y. Elkana. Dordrecht/Boston: Reidel, $197^{8}$.

Hilbert, D. Grundlagen der Geometrie. Stuttgart: Teubner, 1899. (Foundations of geometry. Trad. de L. Unger. La Salle: Open Court, 1971. Les fondements de la géométrie. Trad. de P. Rossier. Paris: Dunod, 1972.)

Houzel, C. Histoire de la théorie des parallèles. In: Rashed, R. (Ed.). Mathématique et philosophie, de l'Antiquité à l'âge classique. Hommage à Jules Vuillemin. Paris: CNRS, 1990. p. 163-79.

. L'apparition de la géométrie non euclidienne. In: Boi, L., Flament, D. \& Salanski, J. M. (Ed.). 1830-1930: un siècle de géométrie, de C. F. Gauss et B. Riemann à H. Poincaré et E. Cartan. Epistémologie, histoire et mathématiques. Berlin: Springer-Verlag, 1992. p. 3-21.

Howard, D. \& StaGhel, J. (Ed.). Einstein and the history of general relativity. Berlin: Birkhauser, 1989.

MAGH, E. On physiological, as distinguished from geometrical space. The Monist, 11, p. 321-50, 1901 . On the psychology and natural development of geometry. The Monist, 12, p. 481-515, 1902. . Space and geometry from the point of view of physical inquiry. The Monist, 14, p. 1-32, 1903. Space and geometry, in the light of physiological, psychological and physical inquiry. Trad. de T. J. McCormack. La Salle: Open Court, 1906.

Minkowski, H. Das Relativitätsprinzip. Annalen der Physik, 47, p. 927-38, 1915 [1907]. (Exposição apresentada em Göttingen em 5/11/1907 e publicada postumamente).

. Raum und Zeit. Physikalische Zeitschrift, 10, p. 104-11, 1909.

Mourão, R. R. de F. Explicando a teoria da relatividade, com apêndice sobre a visita de Einstein ao Brasil. Rio de Janeiro: Ediouro/Tecnoprint, 1987. Apêndice 1: A introdução da teoria da relatividade no Brasil, p. 73-6. Apêndice 2: $O$ eclipse de Sobral, primeira confirmação da relatividade geral, p. 77-86. Apêndice 3: Einstein no Brasil, p. 87-116.

Einstein, de Sobral para o mundo. Sobral: Ed. Uva, 2003.

Moreira, I. \& Videira, A. A. P. (Org.). Einstein e o Brasil. Rio de Janeiro: Editora da UFRJ, 1995.

Mulder, H. L. \& Velde-Schlick, B. F. B. van de. (Ed.). Philosophical papers of Moritz Schlick. Trad. de P. Heath, W. Sellars, H. Feigl \& M. Brodbeck. Dordrecht: Reidel, 1979. 2 v.

PAsch, M. Vorlessungen über neuere Geometrie. Leipzig: Teubner, 1882.

Paty, M. Einstein et la philosophie en France: à propos du séjour de 1922. Cahiers Fundamenta Scientiae, 93, p. 23-411, 1979. (Bulletin de la Société Française de Physique, 35, p. 9-12, jan. 1980.)

. Invention et réception d'une nouvelle théorie et tradition scientifique: le cas de la relativité restreinte et des savants français Poincaré et Langevin. Revista da Sociedade Brasileira de História da Ciência, 2, p. $6-20,1985$.

The scientific reception of relativity en France. In: GLICK, T. (Ed.). The comparative reception of relativity. Dordrecht: Reidel, 1987. p. 113-67.

. Physical geometry and special relativity: Einstein e Poincaré. In: Boi, L.; Flament, D. \& Salanski, J. M. (Ed.). 1830-1930: un siècle de géométrie, de C. F. Gauss et B. Riemann à H. Poincaré et E. Cartan. Epistémologie, histoire et mathématiques. Berlin: Springer-Verlag, 1992.

. Einstein philosophe. La physique comme pratique philosophique. Paris: PUF, 1993.

. Construção matemática e realidade do espaço-tempo da teoria da relatividade. Trad. de S. Chibeni. In: Évora, F. R. (Ed.). Espaço e tempo. Campinas: Unicamp/CLE, 1995· p. 13-4.o. 
Introdução a três textos de Einstein...

A recepção da relatividade no Brasil e a influença das tradições científicas européias. Trad. de A. M. Alves. In: Hamburger, A. I.; Dantas, M. A.; Paty, M. \& Petitjean, P. (Ed.). A ciência nas relações Brasil-França, 1850-1950. São Paulo: Edusp, 1996. p. 143-81.

Poincaré, H. L'espace et la géométrie. Revue de Métaphysique et de Morale, 3, p. 631-4,6, 1895.

On the foundations of geometry. The Monist, 9, p. 1-43, 1898 .

La science et l'hypothèse. Paris: Flammarion, 1902a.

Compte-rendu de Hilbert: Les fondements de la géométrie. Bulletin des Sciences Mathématiques, 26, p. $249^{-} 72,1902$ b.

. L'espace et ses trois dimensions. Revue de Métaphysique et de Morale, 11, p. 281-301; p. 407-29, 1903. La relativité de l'espace. L'année psychologique, 13, p. 1-17, 1907.

Science et méthode. Paris: Flammarion, 1908.

L'espace et le temps. Scientiaï, 25, p. 159-70, 1912a.

Pourquoi l'espace a trois dimensions. Revue de Métaphysique et de Morale, 20, p. 483-504, 1912b.

Rashed, R. (Ed.). Mathématique et philosophie, de l'Antiquité à l'âge classique. Hommage à Jules Vuillemin. Paris: CNRS, 1990.

Reichenbach, H. Relativitätstheorie und Erkenntnis a priori. Berlin: Springer, 1920. (The theory of relativity and a priori knowledge. Trad. de M. Reichenbach. Berkeley: University of California Press, 1965.)

La signification philosophique de la théorie de la relativité. Revue Philosophique de la France et de l'Étranger, 94, p. 5-61, 1922.

.Axiomatik derrelativischen Raum-Zeit-Lehre. Vieweg: Braunschveig, 1924. (Axiomatisation of the theory of relativity. Trad. de M. Reichenbach. Berkeley: University of California Press, 1969.)

. Philosophie der Raum Zeit Lehre. Berlin: de Gruyter, 1928. (The philosophy of space and time. Trad. de M. Reichenbach \& J. Freund. New York: Dover, 1957.)

The philosophical significance of the theory of relativity. In: Schilpp, P. A. (Ed.). Albert Einstein, philosopher-scientist. La Salle: Open Court, 1949. p. 289-311.

. Selected writings. Dordrecht: Reidel, 1978. 2 v.

Riemann, B. Ueber die Hypothesen, welche der Geometrie zugrunde liegen, Abhandlungender königlischen Gessellschaft der Wissenschaften zu Göttingen, 13, 1854. (Texto apresentado em 10/06/1854 à Faculdade de Filosofia de Göttingen.)

Russeld, B. An essay on the foundations of geometry. Cambridge: Cambridge University Press, 1897.

Shields, M. G. Bibliography. In: Schilpp, P. A. (Ed.).Albert Einstein, philosopher-scientist. La Salle: Open Court, 1949. p. 694-760.

Schilpp, P. A. (Ed.). Albert Einstein, philosopher-scientist. La Salle: Open Court, 1949.

Schlick, M. Die philosophische Bedeutung des Relativitätsprinzip. Zeitschrift für Philosophie und philosophische Kritik, 159, p. 129-75, 1915. (The philosophical significance of the principle of relativity. Trad. de P. Heath. In: Mulder, H. L. \& Velde-Schlick, B. F. B. van de. (Ed.). Philosophical papers of Moritz Schlick. Dordrecht: Reidel, 1979. v. 1, p. 153-89.)

Idealität des Raumes, Introjektion und psychophysisches Problem. Vierteljahrsschrift für wissenschaftlische Philosophie und Soziologie, 4.0, p. 230-54, 1916. (Ideality of space, introjection and the psychophysical problem. Trad. de P. Heath. In: Mulder, H. L. \& Velde-Schlick, B. F. B. van de. (Ed.). Philosophical papers of Moritz Schlick. Dordrecht: Reidel, 1979. v. 1, p. 190-206.)

Raum und Zeit in der Gegenwärtigen Physik. Zu Einfuhrung in das Verständnis der Allgemeinen Relativitâtstheorie. Berlin: [s. n.], 1917. (Space and time in contemporary physics. An introduction to the theory of relativity and gravitation. Trad. de H. L. Brose. Oxford: Clarendon Press, 1920.)

Allgemeine Erkenntnislehre. Naturwissenschaftliche Monographien und Lehrbücher I. Berlin: [s. n.], 1918. (General theory of knowledge. Trad. de A. E. Blumberg. New York: Library of Exact Philosophy, 1974.) 
Kritizistische oder empiritische Deutung der neuen Physik. Kant Studien, 26, p. 96-111, 1921a. (Critical or empiricist interpretation of modern physics. Trad. de P. Heath. In: Mulder, H. L. \& VELDESchlick, B. F. B. van de. (Ed.). Philosophical papers of Moritz Schlick. Dordrecht: Reidel, 1979. v. 1, p. $322-34$.

Helmholtz als Erkenntnistheoretiker. In: Helmholtz als Physiker, Physiologe und Philosoph. Karlsruhe: [s. n.], 1921b. p. 29-39. (Trad. inglesa: Mulder, H. L. \& Velde-Schlick, B. F. B. van de. (Ed.). Philosophical papers of Moritz Schlick. Dordrecht: Reidel, 1979. v. 1, p. 335-42.)

Staghel, J.; Klein, M. et al. (Ed.). The collected papers of Albert Einstein. Princeton: Princeton University Press, 1989. v. 2.

Stäckel, P. \& Engel, F. Die Theorie der parallellelinien von Euklid bis Gauss. Eine Urkundensammlung zur Vorgeschichte der Nichteuklidischen Geometrie. Leipzig: Teubner, 1895.

Tolmasquim, A. T. Einstein. O viajante da relatividade na América do Sul. Rio de Janeiro: Vieira \& Lent, 2005 .

Tonetti, R. Philosophy of geometry from Riemann to Poincaré. Dordrecht: Reidel, $197^{8}$. Relativity and geometry. Oxford: Pergamon Press, 1983.

Veronese, G. Fundamenti della geometria a piu dimensioni e a piu specie di unità rettilinee, esposti in forma elementare. Padova: Tipografia del Seminario, 1891.

Vuillemin, J. Poincaré's philosophy of space. Synthese, 24, p. 161-79, 1972.

Westerkampf, F. Evolución de las ciencias en la República Argentina, 1923-1972. Buenos Aires: Sociedad Cientifica Argentina, 1975. Tomo 2: Física.

WeYL, H. Temps, espace, matière. Leçons sur la théorie de la relativité générale. Trad. de G. Juvet \& R. Leroy. Paris: Blanchard, 1922 [1918]. 